Standard Article

J Vet Intern Med 2017;31:505-512

\title{
Clinical Features, Imaging Characteristics, and Long-term Outcome of Dogs with Cranial Meningocele or Meningoencephalocele
}

\author{
K. Lazzerini, R. Gutierrez-Quintana, R. José-López, F. McConnell, R. Gonçalves, J. McMurrough,
} S. De Decker, C. Muir, S.L. Priestnall, L. Mari, F. Stabile, L. De Risio, C. Loeffler, A. Tauro, C. Rusbridge, S. Rodenas, S. Añor, C. de la Fuente, A. Fischer, A. Bruehschwein, J. Penderis, and J. Guevar

\begin{abstract}
Background: The term meningoencephalocele (MEC) describes a herniation of cerebral tissue and meninges through a defect in the cranium, whereas a meningocele (MC) is a herniation of the meninges alone.

Hypothesis/Objectives: To describe the clinical features, magnetic resonance imaging (MRI) characteristics, and outcomes of dogs with cranial MC and MEC.

Animals: Twenty-two client-owned dogs diagnosed with cranial MC or MEC.

Methods: Multicentric retrospective descriptive study. Clinical records of 13 institutions were reviewed. Signalment, clinical history, neurologic findings and MRI characteristics as well as treatment and outcome were recorded and evaluated.

Results: Most affected dogs were presented at a young age (median, 6.5 months; range, 1 month -8 years). The most common presenting complaints were seizures and behavioral abnormalities. Intranasal MEC was more common than parietal MC. Magnetic resonance imaging identified meningeal enhancement of the protruded tissue in $77 \%$ of the cases. Porencephaly was seen in all cases with parietal MC. Cerebrospinal fluid (CSF) analysis identified mild abnormalities in 4 of 11 cases. Surgery was not performed in any affected dog. Seventeen patients were treated medically, and seizures were adequately controlled with anti-epileptic drugs in 10 dogs. Dogs with intranasal MEC and mild neurologic signs had a fair prognosis with medical treatment.

Conclusion and clinical importance: Although uncommon, MC and MEC should be considered as a differential diagnosis in young dogs presenting with seizures or alterations in behavior. Medical treatment is a valid option with a fair prognosis when the neurologic signs are mild.
\end{abstract}

Key words: Cerebral malformation; Cranioschisis; Porencephaly; Seizures.

M alformations of the skull include incomplete closure of the calvaria. This opening of the cranium, termed cranioschisis, is a potential gap in the skull

From the Small Animal Hospital, University of Glasgow, Glasgow, (Lazzerini, Gutierrez-Quintana, José-López, Guevar), Small Animal Teaching Hospital, University of Liverpool, Liverpool, (McConnell, Gonçalves); PetMedics, Worsley, Manchester (McMurrough); Queen Mother Hospital for Animals, Royal Veterinary College, London, (De Decker); Pathology and Pathogen Biology, Royal Veterinary College, Hawkshead Lane Hatfield, Hertfordshire, AL9 7TA, UK (Muir, Priestnall); Animal Health Trust, Newmarket, UK (Mari, Stabile, De Risio); Faculty of Veterinary Medicine, University of Leipzig, Leipzig, Germany (Loeffler); Fitzpatrick Referrals, Eashing, Surrey UK (Tauro, Rusbridge); Hospital Veterinario Valencia Sur, Valencia, (Rodenas); Fundació Hospital Clinic Veterinari, Facultat de Veterinària, Universitat Autònoma de Barcelona, Bellaterra, Barcelona, Spain (Añor, de la Fuente); Ludwig-Maximilian University Munich, Munchen, Germany (Fischer, Bruehschwein); Vet Extra Neurology, Broadleys Veterinary Hospital, Stirling, UK (Penderis).

Preliminary results were presented as oral communication and abstract at the 28th European ESVN-ECVN Congress on September 18.-19., 2015, in Amsterdam, the Netherlands.

Corresponding author: J. Guevar, Small Animal Hospital, College of Medical, Veterinary and Life sciences, University of Glasgow, 464 Bearsden Road, G61 1QH Glasgow; e-mail: Julien.guevar@ gmail.com

Submitted September 14, 2016; Revised October 27, 2016; Accepted November 21, 2016.

Copyright (C) 2017 The Authors. Journal of Veterinary Internal Medicine published by Wiley Periodicals, Inc. on behalf of the American College of Veterinary Internal Medicine.

This is an open access article under the terms of the Creative Commons Attribution-NonCommercial License, which permits use, distribution and reproduction in any medium, provided the original work is properly cited and is not used for commercial purposes.

DOI: 10.1111 /jvim.14638

\begin{tabular}{|c|c|}
\hline \multicolumn{2}{|c|}{ Abbreviations: } \\
\hline AED & anti-epileptic drugs \\
\hline CSF & cerebrospinal fluid \\
\hline $\mathrm{CT}$ & computed tomography \\
\hline FLAIR & fluid-attenuated inversion recovery \\
\hline GRE & gradient recall echo \\
\hline $\mathrm{MC}$ & meningocele \\
\hline MEC & meningoencephalocele \\
\hline MRI & magnetic resonance imaging \\
\hline $\mathrm{T} 2 \mathrm{~W}$ & T2-weighted sequence \\
\hline TNCC & total nucleated cell count \\
\hline
\end{tabular}

through which tissue can protrude. A meningoencephalocele (MEC) is a protrusion of cerebral tissue and meninges through a cranial defect, whereas a meningocele (MC) is a herniation of the meninges only. ${ }^{1}$ The prevalence of cranial MC and MEC in dogs currently is unknown, and there are only 4 case reports described. The reported dogs had different etiologies (likely congenital and acquired after skull fracture), different ages at presentation (neonatal to 6 years old), different localizations of the MC or MEC (1 parietal, 3 intranasal), and variability in the severity of clinical signs and outcomes. ${ }^{2-5}$

In human medicine, the prevalence of congenital MEC is approximately $1 / 40,000$ births. $^{6}$ Severe MECs are diagnosed prenatally or at birth, whereas discrete MECs may only cause clinical signs at an adult age. They are classified according to the anatomic localization of the skull defect. ${ }^{7}$ Fronto-ethmoidal MECs protruding into the nasal cavity are named intranasal MECs. Many patients with intranasal MEC present 
without any neurologic signs, and only with signs of nasal obstruction or rhinorrhea. ${ }^{8}$ If neurologic deficits are observed, they mostly consist of seizures. ${ }^{9}$ The imperative for surgical treatment is strong in all patients. ${ }^{10}$ Outcome is largely dependent on the localization of the skull defect and the content of the herniated sac (infants with a large sac have a worse outcome). ${ }^{11}$

The aim of our study was to expand the current knowledge of MECs and MCs by reporting the clinical presentation, imaging features, treatment, and outcome of a large case series of canine cases. We hypothesized that, although a MEC may be an epileptic focus, dogs with mild-to-moderate neurologic signs may respond to medical management alone.

\section{Material and Methods}

\section{Study Design}

The clinical records of dogs presented to several neurology referral centers between 2006 and 2016 and diagnosed with cranial MC or MEC were retrospectively analyzed. Ethical approval was granted from the University of Glasgow for this study (Ref 31a/ $15)$.

Inclusion criteria included complete neurologic examination, diagnosis of cranial MC or MEC, MRI of the brain, and sufficient clinical information available for review.

The following information was recorded for each case: signalment, presenting complaint, duration of clinical signs, seizure type if applicable, and findings on physical and complete neurologic examinations at presentation. Results of MRI and CSF examination also were evaluated. Treatment, outcome (duration of follow-up period, frequency of seizures, reason for euthanasia), and necropsy results (if applicable) were recorded. Dogs were classified as responders if they experienced $\mathrm{a} \geq 50 \%$ decrease in seizure frequency from baseline or absence of seizures, ${ }^{12}$ when data were available, or as improved signs and controlled seizures, if data were insufficient to determine whether a $50 \%$ decrease in seizure frequency was achieved, but owners reported that seizure frequency was decreased and quality of life was adequate.

Magnetic resonance imaging was performed with scanners of variable field strength $(0.2 \mathrm{~T}-3.0 \mathrm{~T})$. Anesthetic protocols and patient positioning varied according to the neurology center. All studies included spin-echo or turbo spin-echo T2-weighted sagittal and transverse views, both pre- and postcontrast (gadopentetate dimeglumine) spin-echo or turbo spin-echo T1-weighted transverse views and T2-weighted fluid-attenuation inversion recovery (FLAIR) transverse views. Some of these studies included short tau inversion recovery sequences, gradient recall echo (GRE) transverse views, spin-echo or turbo spin-echo T2-weighted dorsal views, pre- and postcontrast spin-echo or turbo spin-echo T1weighted dorsal and sagittal views and FLAIR dorsal views.

One board-certified veterinary neurologist (RGQ), blinded to signalment and neurologic examination, retrospectively reviewed and described the MRI findings for each case. The following criteria were recorded: affected bone, protrusion of meninges alone or meninges and cerebral tissue, hyperintensity of protruded tissue on T2-weighted images compared to normal gray matter, presence of fluid in the meningeal sac, presence of a signal void on GRE sequences in the protruding tissue (whenever sequence was available), presence of meningeal enhancement of the protruding meninges after contrast administration, presence of other intracranial MRI findings and concurrent malformations, asymmetry of nonprotruded brain tissue (characterized by cranial displacement of the lateral ventricle, distortion of the corpus callosum, or both), and presence of a unilateral or bilateral protrusion.

For the intranasal MEC, the amount of protruding tissue was subjectively classified with a 3-point grading scale (mild, moderate, and severe) dependent on the amount of tissue protruding through the cranial defect on the basis of dorsal T2-weighted images at the level of maximum protrusion as a consensus between 2 observers, RGQ and 1 European College of Veterinary Neurology (ECVN) residency-trained neurologist (JG). Dorsal T1weighted images were used if $\mathrm{T} 2$-weighted images were not available. Subjective assessment, which is likely to be the method employed in a clinical setting to determine MEC size, was compared with a quantitative measurement of MEC volume. To allow comparison among dogs of different sizes, volume ratio between protruded brain tissue and intracalvarial brain tissue was calculated. Three-dimensional visualization of the normal brain and MEC was performed in all cases with intranasal MEC by the software Amira ${ }^{\mathrm{a}}$ based on T2-weighted transverse images (Fig. S1). Volume of MEC and brain was measured by Mud box ${ }^{\mathrm{b}}$ based on the 3-dimensional reconstruction. Observers were blinded to the clinical status.

The data were not normally distributed; therefore, descriptive statistics using median and range were employed. The nonparametric Kruskal-Wallis test was used to compare the volume ratio between MEC and intracalvarial brain tissue in the 3 groups determined by subjective assessment (mild, moderate, and severe). Mann-Whitney U-test was used as posthoc analysis to determine differences between each group, by the statistical software SPSS

\section{Results \\ Clinical Features}

Twenty-two dogs diagnosed with cranial MC or MEC at 13 institutions met the inclusion criteria. The age at time of diagnosis varied from 1 month to 8 years (median, 6.5 months). The following breeds were represented: Jack Russell Terrier (3), Border Collie (2), Labrador Retriever (2), Chihuahua (1), Weimaraner (1), Toy Poodle (1), Malinois (1), Cocker Spaniel (1), Boxer (1), Pointer (1), Cavalier King Charles Spaniel (1), English Springer Spaniel (1), Welsh sheepdog (1), miniature Dachshund (1), and crossbreed dogs (4). Eleven of the dogs were male (4 neutered), and 11 were female (2 spayed).

The most common presenting clinical signs were epileptic seizures $(\mathrm{n}=17,77 \%)$ and abnormal behavior $(\mathrm{n}=7,31 \%)$. Median duration of clinical signs before presentation was 30 days (range, 12 hours -1 year). In $1 \mathrm{dog}$ (case 10), the meningoencephalocele was considered an incidental diagnostic imaging finding. The signalment and clinical signs of the patients are described in Table S1.

Information regarding seizure characteristics was available in 16 of 17 cases. Only generalized seizures were present in 10 dogs $(63 \%), 1$ dog experienced focal seizures $(6 \%)$, and $5 \operatorname{dogs}(31 \%)$ had both focal and generalized seizures. Abnormal behavior was seen in 7 dogs $(30 \%)$ and included aggressiveness (2), compulsive 
behavior (4), hyperactivity (2), intermittent yelping (2), and star-gazing or fly-catching behavior (1).

Physical examination was normal in 17 dogs (77\%). Two dogs had an open fontanel, 1 dog had a domeshaped head, 1 dog had a facial deformity and right nasolacrimal duct obstruction secondary to a dog bite, and 1 dog had nasal discharge (suspected CSF rhinorrhea). Another dog developed suspected CSF rhinorrhea after diagnosis. Neurologic examination was abnormal in 16 of $22 \operatorname{dogs}(73 \%)$. One dog had a concurrent neurologic disease (lymphoma). Abnormalities in the neurologic examination in dogs without concurrent disease $(\mathrm{n}=15)$ consisted of decreased mentation $(n=2,13 \%)$, circling $(n=5,33 \%)$, vestibular ataxia $(\mathrm{n}=1,7 \%$; parietal meningocele), cerebellar ataxia $(\mathrm{n}=1,7 \%$; frontal and parietal meningocele), proprioceptive deficits (lateralized $[n=9,60 \%]$, bilateral symmetric $[\mathrm{n}=2,13 \%])$, absent or decreased menace response (unilateral $[\mathrm{n}=5,33 \%]$, bilateral $[\mathrm{n}=4$, $27 \%])$, unilateral decreased nasal sensation $(\mathrm{n}=3$, $20 \%)$, strabismus $(\mathrm{n}=2,14 \%)$, intermittent positional nystagmus $(\mathrm{n}=1,7 \%$; parietal meningocele), and hyperesthesia of the head $(\mathrm{n}=2,13 \%)$ and cervical spine ( $\mathrm{n}=1,7 \%$; parietal meningocele). Abnormal neurologic examination findings are described in Tables $\mathrm{S} 2 \mathrm{a}$ and S2b.

In dogs with abnormal neurologic examination findings $(73 \%)$, the neuro-anatomic localization was to the forebrain (bilateral in 3 cases [19\%], lateralized in 9 cases $[56 \%]$ ), and to the cerebellum in 1 case (frontal and parietal meningocele). Multifocal signs were seen in 2 dogs $(13 \%$; 1 with concurrent lymphoma and 1 with syringomyelia), and in 1 , the lesion was localized to caudal cervical region (diagnosed with a presumably unrelated cervical lesion consistent with focal myositis or neuritis).

\section{Magnetic Resonance Imaging Findings}

Seventeen dogs were diagnosed with an intranasal MEC (73\%; Fig 1). The ethmoidal bone was affected in 15 dogs $(68 \%)$. The frontal bone was affected in 2 dogs. The parietal bone was affected in 6 of 22 dogs $(27 \%)$. One dog had a concurrent frontal MEC and parietal MC (Fig 2). One dog was diagnosed with a frontal MEC, which did not protrude into the sinus but laterally under the temporal muscle (Fig 3).

In all dogs in which the ethmoidal bone and frontal bone were affected, neuronal tissue protruded through the bone defect. Meninges alone appeared to protrude through parietal bone defects. The protruding brain tissue was hyperintense on $\mathrm{T} 2 \mathrm{w}$ sequences in 14 of 17 cases, with presence of fluid (isointense compared to CSF signal) in the meningeal sac in 6 of 17 dogs, and evidence of hemorrhage in 6 of 13 dogs (GRE sequences performed in 13 dogs). After IV administration of gadopentetate dimeglumine, meningeal enhancement of the protruding tissue was seen in 17 of 22 dogs on T1w images. Concurrent malformations were seen in 3 of 17 dogs with intranasal lesions. Two dogs had severe ethmoidal MECs with most likely compensatory hydrocephalus ex vacuo. Concurrent malformations were seen in all dogs with parietal meningocele (porencephaly in 4 dogs, supracollicular fluid accumulation in $1 \mathrm{dog}$ ). In the dog with frontal MEC and parietal MC, several concurrent malformations were detected: hydrocephalus ex vacuo, possible cerebellar hypoplasia, dilatation of the olfactory recess, and porencephaly. The MEC or cranial MC was

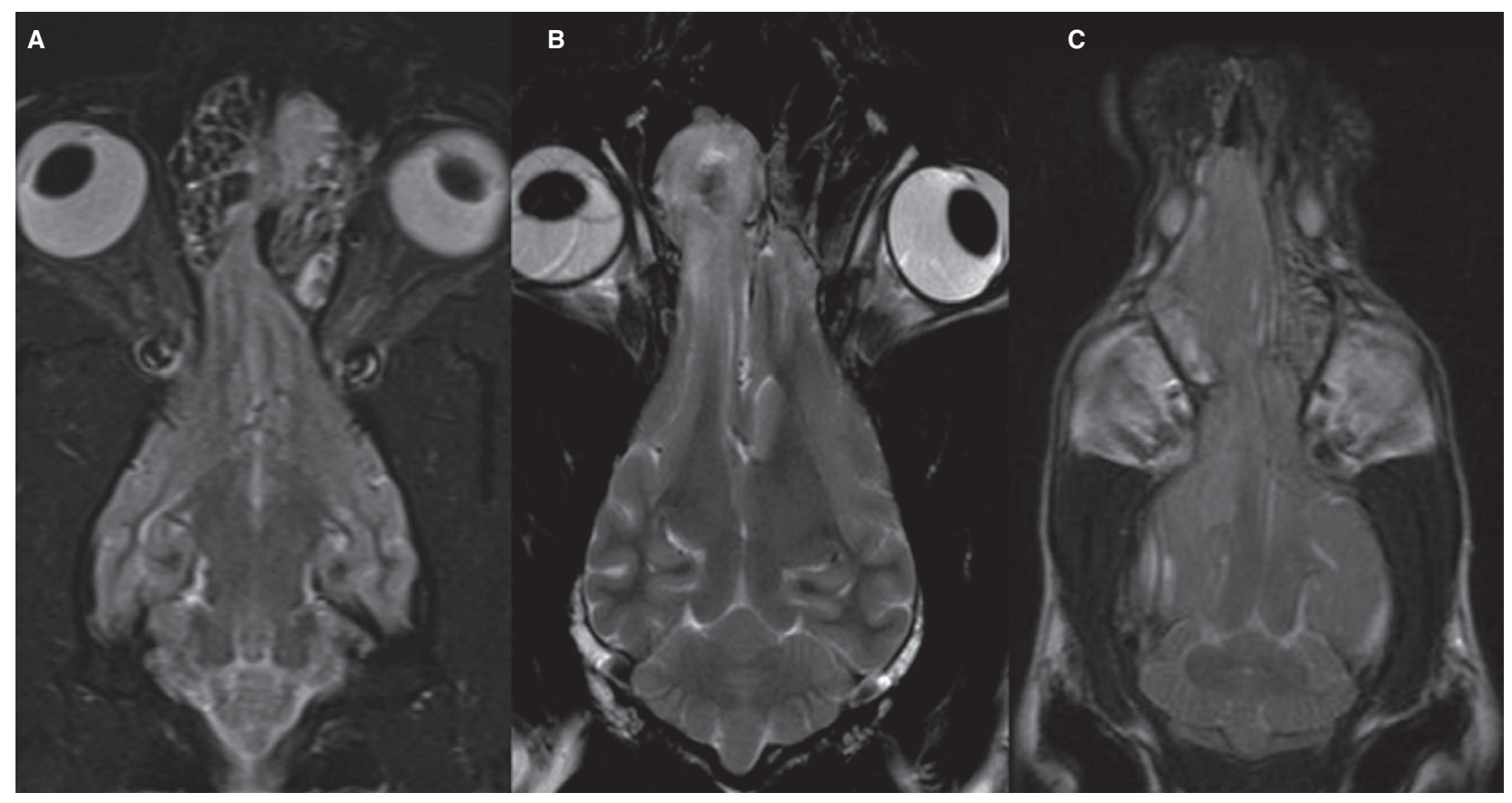

Fig 1. Dorsal magnetic resonance images of the brain and nose of dogs with a mild (A), moderate (B), and severe (C) unilateral ethmoidal meningoencephalocele. Short tau inversion recovery (A), T2-weighted sequence (B,C). 


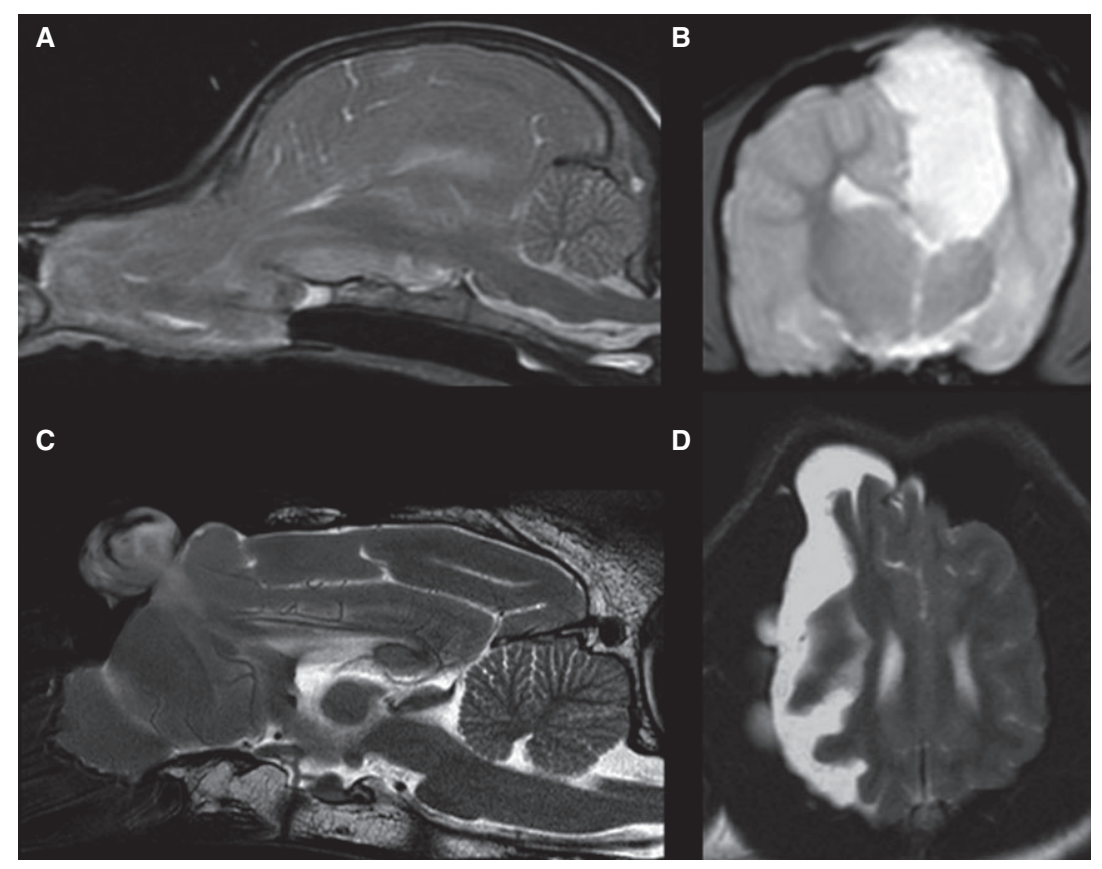

Fig 2. T2-weighted magnetic resonance images of the brain of dogs with meningoencephalocele and meningocele. A: midsagittal view of the brain of a dog with a severe intranasal ethmoidal meningoencephalocele. B: transverse view of the brain at the level of the thalamus depicting a unilateral parietal meningocele with associated porencephaly and asymmetry of the remaining brain tissue. C: midsagittal view of the brain of a dog with frontal meningoencephalocele into the frontal sinus. D: dorsal view of the brain and nose of a dog with unilateral parietal meningocele and intranasal frontal meningoencephalocele.

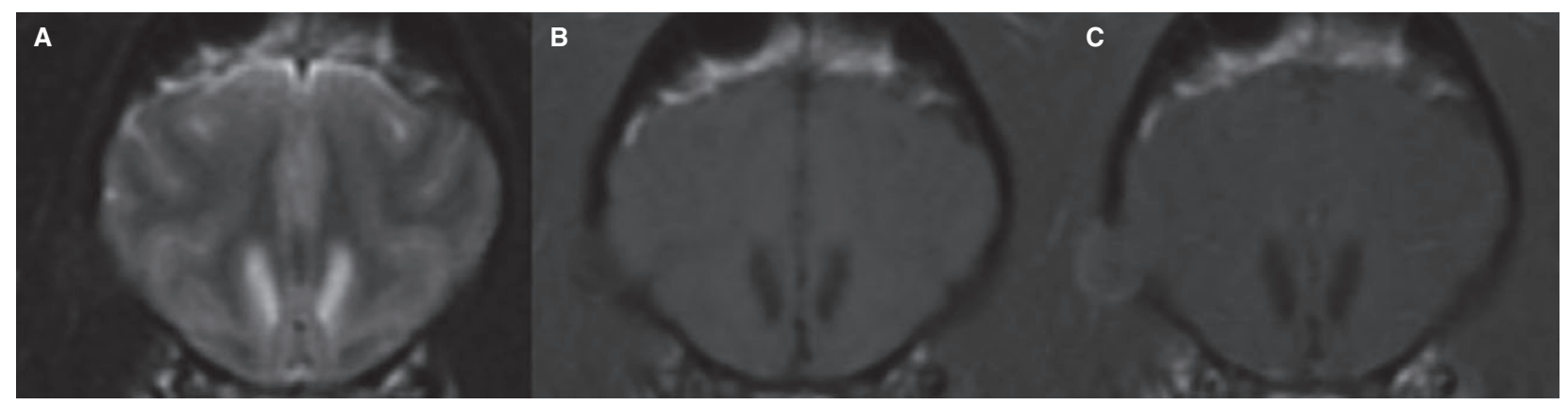

Fig 3. Transverse magnetic resonance imaging sequences of the forebrain of a dog with frontal meningoencephalocele, T2-weighted sequence (A), T1-weighted sequence before contrast (B) and after intravenous contrast (C). Note the contrast enhancement of the protruded meninges.

unilateral in 19 of 22 dogs. The remaining brain tissue showed asymmetry in 12 of 22 dogs. The MRI findings are summarized in Table S3.

Subjective assessment of intranasal MEC size was performed in 16 dogs (dog 22 was excluded based on concurrent parietal MC). The MEC was considered mild in 4 dogs, moderate in $7 \mathrm{dogs}$, and severe in 5 dogs. Overall, the calculated volume ratio between MEC and intracalvarial brain was significantly different in the 3 groups identified by subjective assessment of the amount of protruded tissue (mild, moderate, and severe; $P=.009$ ). Individual comparison among groups identified a significant difference between the groups moderate and severe $(P=.028)$ and the groups mild and severe $(P=.014)$. The difference between the groups mild and moderate was not statistically significant $(P=.059$; Fig S2).

\section{Results of CSF Analysis}

Cerebrospinal fluid analysis was performed in 11 dogs. It was normal in 6 dogs $(55 \%)$ and identified abnormalities in 5 dogs $(45 \%)$. One dog was diagnosed with a concurrent condition (case 10, lymphoma). The CSF analysis disclosed mild changes in 4 samples (increased eosinophil count in $1 \mathrm{dog}$, mixed pleocytosis in 2 dogs, mildly increased protein concentration in 1 dog). All dogs with abnormal CSF and without concurrent disease had intranasal MECs. Results of CSF analysis are summarized in Table $\mathbf{S} 4$. 


\section{Outcome}

Eleven dogs were alive $(50 \%)$, and 9 dogs were dead $(41 \%)$ at the time of writing. The outcome is unknown in 2 cases. Four dogs were euthanized shortly after diagnosis because of the severity of their clinical signs $(18 \%)$. One dog with parietal MC did not receive any medication (alive at the last follow-up 8 weeks after diagnosis). Medical treatment was started in 17 dogs. Treatment consisted of anti-epileptic medication in 13 of 17 dogs (phenobarbital $[\mathrm{n}=11]$, imepitoin $[\mathrm{n}=3$ ], levetiracetam $[\mathrm{n}=4]$, potassium bromide $[\mathrm{n}=2]$, combination of 2 anti-epileptic drugs (AED) or more $[n=5])$. Seven dogs were treated with a course of prednisolone (variable anti-inflammatory doses, tapered over a few weeks). Three dogs with inflammatory CSF findings were treated with a course of antibiotics (amoxicillin-clavulanic acid, metronidazole).

The follow-up period ranged from 2 months to 4 years, with a median of 365 days. In 11 dogs, the seizures were controlled by anti-epileptic treatment at the last follow-up. Five dogs were considered responders (2 dogs are seizure-free, $3 \mathrm{dogs}$ had a seizure reduction of $\geq 50 \%$ ), and in 6 dogs, an improvement of seizure frequency was noted but follow-up data did not specify whether seizure frequency was decreased by $\geq 50 \%$. In 3 dogs, seizures were initially controlled by AED for a duration of 1 and 4 years (unknown duration in $1 \mathrm{dog}$ ), but they were euthanized at a later stage because of uncontrolled seizures.

All 4 dogs in which the intranasal MEC was classified as mild with MRI were alive at final follow-up (followup period, 548 days - 1095 days; median, 730 days). In 7 dogs, the MECs were considered moderate. Three of these dogs were euthanized (1 immediately after diagnosis, 2 at a later stage because of uncontrolled seizures), and 4 were still alive at the last follow-up. The MECs were considered severe in 5 dogs, 4 of which were euthanized ( 3 shortly after diagnosis, 1 was euthanized 1 year after diagnosis because of uncontrolled seizures). Treatment and outcome of all dogs are summarized in Tables S5a and S5b and in Figure S7.

\section{Gross and Histopathologic Findings}

Necropsy was performed on 2 dogs. One dog (dog 1) was euthanized shortly after diagnosis. One $\operatorname{dog}(\operatorname{dog} 6)$ was treated medically for 4 years and euthanized at 12 years of age because of recurrent seizures.

In both cases, the olfactory lobe protruded into the nasal cavity through a closure defect in the cribriform plate. The herniated olfactory lobe was composed of necrotic neuronal tissue overlaid by a thick (approximately $1 \mathrm{~mm}$ ) layer of mature collagenous connective tissue (fibrosed dura), which in turn was covered by a single layer of respiratory epithelium. Occasionally expanding the dura were deposits of mineral (dystrophic mineralization) and the dura blended with firm adherence to the remaining cribriform plate (dog 6). There was a distinct, approximately $1 \mathrm{~cm}$, gap between the herniated olfactory lobe and the dorsal nasal conchae (dog 6). Infiltrating the neuropil of the olfactory lobe were frequent oligodendrocytes and astrocytes (gliosis) and proliferations of immature fibrovascular connective tissue (neovascularization). Frequent pools of hemorrhage were present throughout (Fig 4).

The right frontal lobe adjacent to the protruded olfactory lobe was chronically inflamed and edematous, and in $\operatorname{dog} 6$, these changes caused compression of the
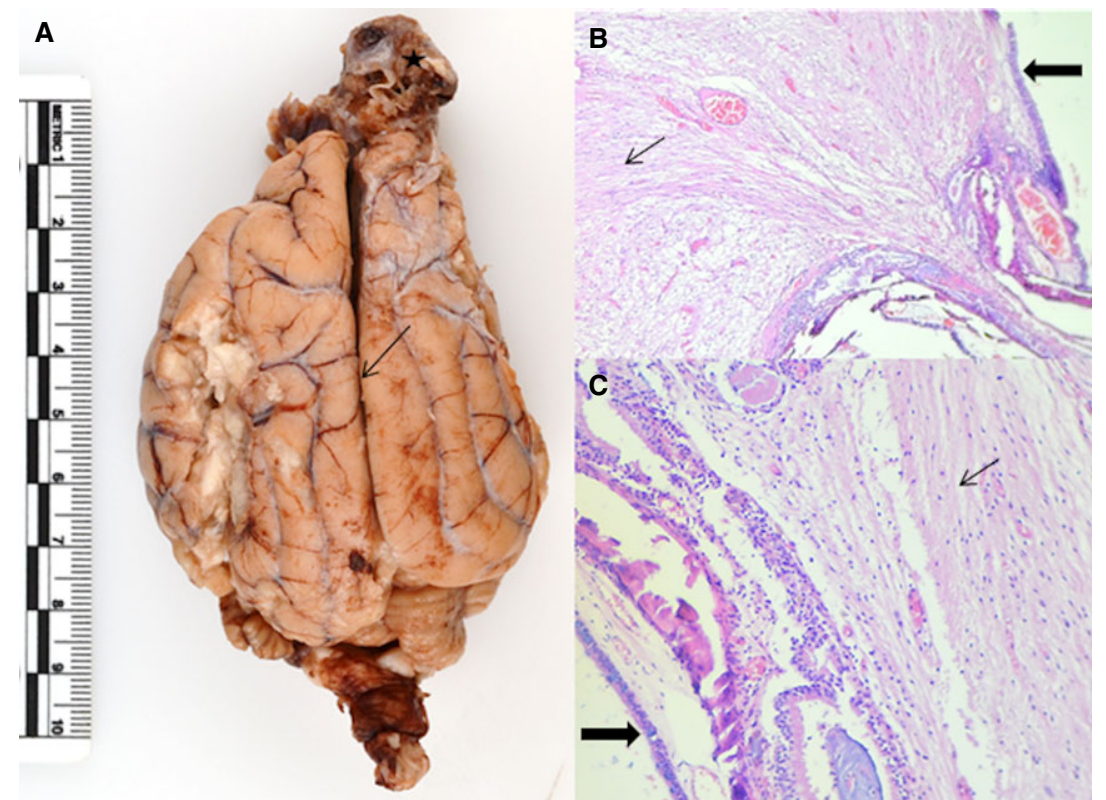

Fig 4. A: Unilateral ethmoidal meningoencephalocele (Arrow: Pronounced midline shift// Star: Abundant hemorrhage within the herniated olfactory bulb). B: Herniated olfactory bulb (Large arrow: Respiratory epithelium/// Thin arrow: Neuropil// ( $\times 40$ Hematoxylin and eosin) C: Herniated olfactory bulb ((Large arrow: Respiratory epithelium/// Thin arrow: Neuropil//) ( $\times 200$ Hematoxylin and eosin). 
left frontal lobe and a pronounced midline shift. Underlying both lateral ventricles were small irregular proliferations of fibrovascular connective tissue (neovascularization).

\section{Discussion}

We describe 22 dogs with cranial MC, MEC, or both, a rare condition with variable outcome. An MEC typically forms as a result of a congenital defect during the closure of the neural tube but can also arise after trauma. ${ }^{4}$ In human medicine, MECs developing as a result of chronically increased intracranial pressure ${ }^{13}$ or postsurgery ${ }^{14}$ have been described. Congenital MCs and MECs have been reported in humans and various domestic animals. ${ }^{15,16}$ A hereditary predisposition has been shown in Burmese cats, ${ }^{16}$ but teratogenic factors and nutritional deficiencies may play a role in the development of such malformations. ${ }^{17,18}$ Based on the small number of cases collected throughout Europe in this study, it appears to be a sporadically occurring disease, that might be underdiagnosed because diagnosis requires advanced imaging and some dogs may not have any neurologic signs, as occurred in the dog diagnosed with lymphoma and an incidental MEC. Early head trauma could be a predisposing factor, as occurred in at least 1 of the cases.

The MECs are classified according to the anatomic localization of the skull defect. ${ }^{7}$ In the human literature, the term encephalocele defines a cephalic hernia through a skull defect and includes both MEC and MC. ${ }^{19}$ The occipital region is the most common site of protrusion in humans in Western regions, ${ }^{20}$ whereas, for unknown reasons, fronto-ethmoidal encephaloceles are more common in South-East Asia ( $1: 5,000$ live births $\left.{ }^{21}\right)$. Most of the cases presented here were ethmoidal (15 of 22, 68\%) and intranasal (16 of 22, 73\%). This finding does not necessarily reflect the occurrence within the canine population and may be explained by the fact that puppies with severely debilitating diseases and obvious malformations are likely to be euthanized at an early age. In humans, the localization of the encephalocele has an important impact on the prognosis for the patient, ${ }^{22}$ caudally located lesions being associated with worse neurologic signs and outcomes than rostrally located lesions. ${ }^{19}$

The protruded parenchyma was heterogeneously hyperintense on T2w and FLAIR images in 14 dogs. These findings could be consistent with gliosis of cerebral tissue, tissue inflammation, or edema. In 2 dogs, histology of the protruded brain tissue indicated that this tissue exhibited a paucity of neurons, multifocal new vessel formation, and malacia. This observation correlates with the results described in previous case reports that identified gliosis and areas of malacia. ${ }^{3}$ In humans, gliosis of the protruded tissue is a common finding. ${ }^{23}$ In some cases, histopathology of the MEC in humans showed fibrosis and chronic inflammation. ${ }^{24,25}$

Magnetic resonance imaging indicated that the protruded meninges were associated with contrast enhancement on $\mathrm{T} 1 \mathrm{w}$ postcontrast images in 17 of 22 cases. This finding could be consistent with focal meningitis or low
CSF pressure. Cerebrospinal fluid analysis was performed in 12 dogs, and abnormal CSF findings did not correlate with meningeal enhancement on MRI. In 10 dogs with meningeal enhancement, CSF was normal in 6 dogs and showed abnormalities in 4 dogs. In 1 dog, histologic examination of the herniated tissue indicated chronic meningitis, but meningeal enhancement of the protruded tissue was not detected on MRI. Magnetic resonance imaging had low sensitivity for diagnosis of meningeal pathology in dogs when compared to histologic findings. ${ }^{26}$ In 1 study, meningeal enhancement was identified in only $28 \%$ of 25 dogs with inflammatory $\mathrm{CSF}^{27}$ In human medicine, meningeal enhancement of pachymeninges on MRI, as well as venous sinus engorgement and subdural effusions, has been associated with intracranial hypotension. ${ }^{28,29}$ The same pathological processes could explain the meningeal enhancement in the patients presented here, because the brain is no longer contained within an intact cranial vault, which may impair CSF and intracranial volume homeostasis (Monro-Kellie doctrine) in patients with cranial MC or MEC.

Seizures were the main presenting neurologic sign in most of the dogs with intranasal lesions. All dogs with focal seizures had unilateral intranasal MECs. However, 8 dogs with unilateral intranasal MECs were reported to only display generalized seizures. Only 1 dog with parietal MC presented with seizures. This observation reflects the neuro-anatomic localization of the lesion, but seizure characteristics do not seem to be a reliable indicator of the localization of the defect. In humans, MECs are considered epileptic foci. ${ }^{30}$ This theory is supported by the fact that surgical repair of MECs generally results in elimination of seizures. ${ }^{5,30,31}$ Several hypotheses have been formulated regarding the pathophysiology of seizures in encephalocele patients, including irritative traction or herniation of involved cortex or hemorrhage, malacia, degeneration white matter, and inflammatory cell infiltrates contributing to abnormal excitability of cortical neurons. ${ }^{3}$ Magnetic resonance imaging and histopathology results in this study confirmed that the herniated tissue shows signs of inflammation and hemorrhage.

Six dogs with intranasal MECs had normal neurologic examination findings. Because even dogs with severe malformation may not show any deficits in neurologic examination, MEC must be considered a differential diagnosis for young dogs presenting with seizures and unremarkable interictal neurologic examination.

Seven dogs had asymmetrical neurologic deficits. The MRI images of 6 of these dogs showed asymmetry of the intracalvarial part of the cerebrum, which was confirmed histopathologically in $\operatorname{dog} 6$. This phenomenon has been described in humans with MEC. ${ }^{32}$ In addition to mechanical traction by the herniated tissue and decreased blood supply to specific areas, ${ }^{33} \mathrm{CSF}$ flow obstruction seems to play a role in the pathogenesis of secondary malformations. ${ }^{32}$

In humans, the most common concurrent malformations found on MRI in patients with MEC were 
hydrocephalus (15\%) and corpus callosum agenesis $(7 \%),{ }^{34}$ with a direct relationship between the number of intracranial abnormalities and the amount of herniated brain. ${ }^{32}$ Agenesis of the corpus callosum has been described in a cat with midline parietal $\mathrm{MEC}^{35}$ but not in any of the dogs presented here. Interestingly, in this study, porencephaly was seen in all dogs with parietal MEC. The clinical and MRI characteristics of porencephaly have been described previously in dogs, some of which had evidence of parietal MC. ${ }^{36,37}$ Additional studies are necessary to determine whether a causal relationship exists between these conditions.

In human medicine, surgical intervention is the treatment of choice, based on the potential for recurrent meningitis, brain damage from herniation, and refractory seizures. ${ }^{38}$ Seizures stopped after surgical intervention in 1 affected dog. ${ }^{5}$ However, in our study, 10 of 17 of dogs presented with seizures responded well to medical treatment with AED. Medical management seems to be a viable treatment option for dogs with only mild neurologic signs. Additional studies examining the longterm outcome of dogs treated medically are required to assess the risk for ascending meningitis in affected dogs. The risk for ascending infection is an important indication for surgery in humans. Therefore, this risk needs to be weighed against the invasiveness of surgical treatment individually until additional studies are available in veterinary medicine. Surgery may be considered in dogs with CSF rhinorrhea or in those with refractory seizures, although the outcome after surgery needs to be evaluated in more cases to assess its benefits.

Case series in human medicine have shown that the size and content of the protruding meningeal sac has an impact on prognosis. ${ }^{11}$ At the last follow-up, all 4 dogs subjectively categorized as mild, but only 4 of 7 dogs with moderate MEC and 1 of 5 dog with severe MEC were still alive. The size of the MEC as well as neurologic signs may have played a role in the decisionmaking process about euthanasia. Subjective assessment based on MRI seems to be a reliable tool to determine MEC size, but additional studies with larger numbers of cases will be necessary to confirm this observation.

The limitations of our study are inherent in its retrospective multicenter nature. The distinction between presumed acquired and congenital MEC or MC could not be made in all cases based on historical information, because information concerning the first weeks of life was limited. The number of parietal MC was limited, and the imaging studies did not follow a specified protocol. Furthermore, treatment protocol was at the clinician's discretion. Follow-up periods were variable, and follow-up did not always include a neurologic examination. Unfortunately, prospective evaluation of an acceptable number of cases is difficult based on the rarity of this condition.

\section{Conclusion}

Although uncommon, MEC should be considered as a differential diagnosis in young dogs presenting with seizures or alterations in behavior. Medical treatment is a valid option with a fair prognosis when the MEC is mild.

\section{Footnotes}

a Amira 5.3.3, Visage Imaging and ZIB, Germany
${ }^{\mathrm{b}}$ Mud box 2016, Autodesk Inc.
${ }^{\mathrm{c}}$ SPSS 17.0 for Windows, SPSS Inc.

\section{Acknowledgments}

We thank Ed Ives and Sue Fitzmaurice at Anderson Moores Veterinary Specialists, Winchester, for submitting the information of case 21 and Hazel Raffan for helping with $3 \mathrm{D}$ reconstruction.

Conflict of Interest Declaration: Authors declare no conflict of interest.

Off-label Antimicrobial Declaration: Authors declare no off-label use of antimicrobials.

\section{References}

1. David DJ. Cephaloceles: Classification, pathology, and management-a review. J Craniofac Surg 1993;4:192-202.

2. Parker AJ, Cusick PK. Meningoencephalocele in a dog (a case history). Vet Med Small Anim Clin 1974;69:206-207.

3. Jeffery N. Ethmoidal encephalocoele associated with seizures in a puppy. J Small Anim Pract 2005;46:89-92.

4. Rosenblatt AJ, Scrivani PV, Caserto BG, et al. Imaging diagnosis-meningoencephalitis secondary to suppurative rhinitis and meningoencephalocele infection in a dog. Vet Radiol Ultrasound 2014;55:614-619.

5. Martle VA, Caemaert J, Tshamala M, et al. Surgical treatment of a canine intranasal meningoencephalocele. Vet Surg 2009;38:515-519.

6. Mahapatra AK, Suri A. Anterior encephaloceles: A study of 92 cases. Pediatr Neurosurg 2002;36:113-118.

7. Suwanwela C, Suwanwela N. A morphological classification of sincipital encephalomeningoceles. J Neurosurg 1972;36:201-211.

8. Kohrmann M, Schellinger PD, Wetter A, Hahnel S. Nasal meningoencephalocele, an unusual cause for recurrent meningitis. Case report and review of the literature. J Neurol 2007;254:259-260.

9. Fountas KN, Smith JR, Jenkins PD, Murro AM. Spontaneous motor cortex encephalocele presenting with simple partial seizures and progressive hemiparesis. Case report and review of the literature. Neurosurg Focus 2005;19:E10.

10. Thompson DN. Postnatal management and outcome for neural tube defects including spina bifida and encephalocoeles. Prenat Diagn 2009;29:412-419.

11. Simpson DA, David DJ, White J. Cephaloceles: Treatment, outcome, and antenatal diagnosis. Neurosurgery 1984;15:14-21.

12. Podell M, Volk HA, Berendt M, et al. 2015 ACVIM Small Animal Consensus Statement on Seizure Management in Dogs. J Vet Intern Med 2016;30:477-490.

13. Kenning TJ, Willcox TO, Artz GJ, et al. Surgical management of temporal meningoencephaloceles, cerebrospinal fluid leaks, and intracranial hypertension: Treatment paradigm and outcomes. Neurosurg Focus 2012;32:E6.

14. Neely JG, Kuhn JR. Diagnosis and treatment of iatrogenic cerebrospinal fluid leak and brain herniation during or following mastoidectomy. Laryngoscope 1985;95:1299-1300. 
15. Cho IC, Park YS, Yoo JG, et al. Two cases of meningocele and meningoencephalocele in Jeju native pigs. BMC Vet Res 2015;11:89.

16. Sponenberg DP, Graf-Webster E. Hereditary meningoencephalocele in Burmese cats. J Hered 1986;77:60.

17. Webster WS, Germain MA, Edwards MJ. The induction of microphthalmia, encephalocele, and other head defects following hyperthermia during the gastrulation process in the rat. Teratology 1985;31:73-82.

18. Scott FW, LaHunta A, Schultz RD, et al. Teratogenesis in cats associated with griseofulvin therapy. Teratology 1975;11:7986.

19. Mealey J Jr, Dzenitis AJ, Hockey AA. The prognosis of encephaloceles. J Neurosurg 1970;32:209-218.

20. Raja RA, Qureshi AA, Memon AR, et al. Pattern of encephaloceles: A case series. J Ayub Med Coll Abbottabad 2008;20:125-128.

21. Suwanwela C. Geographical distribution of frontoethmoidal encephalomeningocele. Br J Prev Soc Med 1972;26: 193-198.

22. David DJ, Proudman TW. Cephaloceles: Classification, pathology, and management. World J Surg 1989;13:349-357.

23. Rapport RL 2nd, Dunn RC Jr, Alhady F. Anterior encephalocele. J Neurosurg 1981;54:213-219.

24. Moore PM. Intranasal encephalomeningocele report of a case. Laryngoscope 1952;62:659-677.

25. Kumar KK, Ganapathy K, Sumathi V, et al. Adult intranasal meningoencephalocele presenting as a nasal polyp. J Clin Neurosci 2005;12:594-596.

26. Keenihan EK, Summers BA, David FH, Lamb CR. Canine meningeal disease: Associations between magnetic resonance imaging signs and histologic findings. Vet Radiol Ultrasound 2013;54:504-515.

27. Lamb CR, Croson PJ, Cappello R, Cherubini GB. Magnetic resonance imaging findings in 25 dogs with inflammatory cerebrospinal fluid. Vet Radiol Ultrasound 2005;46:17-22.

28. Sable SG, Ramadan NM. Meningeal enhancement and low CSF pressure headache. An MRI study. Cephalalgia 1991;11:275-276.

29. Pannullo SC, Reich JB, Krol G, et al. MRI changes in intracranial hypotension. Neurology 1993;43:919-926.

30. Byrne RW, Smith AP, Roh D, Kanner A. Occult middle fossa encephaloceles in patients with temporal lobe epilepsy. World Neurosurg 2010;73:541-546.

31. Faulkner HJ, Sandeman DR, Love S, et al. Epilepsy surgery for refractory epilepsy due to encephalocele: A case report and review of the literature. Epileptic Disord 2010;12:160-166.

32. Sibayan RQ, Racelis LC, Domingo ML. Intracranial abnormalities and ventricular patterns associated with meningoencephaloceles. J Clin Neurosci 1995;2:45-47.

33. Suwanwela C, Hongsaprabhas C. Fronto-ethmoidal encephalomeningocele. J Neurosurg 1966;25:172-182.
34. Mahapatra AK, Agrawal D. Anterior encephaloceles: A series of 103 cases over 32 years. J Clin Neurosci 2006;13: 536-539.

35. Dewey CW, Brewer DM, Cautela MA, et al. Surgical treatment of a meningoencephalocele in a cat. Vet Surg 2011;40:473-476.

36. Schmidt MJ, Klumpp S, Amort K, et al. Porencephaly in dogs and cats: Magnetic resonance imaging findings and clinical signs. Vet Radiol Ultrasound 2012;53:142-149.

37. Davies ES, Volk HA, Behr S, et al. Porencephaly and hydranencephaly in six dogs. Vet Rec 2012;170:179.

38. Woodworth BA, Schlosser RJ, Faust RA, Bolger WE. Evolutions in the management of congenital intranasal skull base defects. Arch Otolaryngol Head Neck Surg 2004;130:1283-1288.

\section{Supporting Information}

Additional Supporting Information may be found online in the supporting information tab for this article:

Figure S1. Three-dimensional reconstruction of the intracalvarial brain (purple) and intranasal meningoencephalocele (green) based on transverse, sagittal and dorsal T2-weighted magnetic resonance imaging sequences.

Figure S2. Box-and-whisker plot diagram representing the subjective grading of MEC compared with ratio between MEC volume and intracalvarial brain volume in dogs with intranasal MEC.

Figure S3. Treatment and outcome in all cases.

Table S1. Overview of the signalment, presenting complaint and diagnosis for all cases of MC/MEC.

Table S2a. Neurological signs and localization of intranasal (ethmoidal and frontal) MEC after MRI.

Table S2b. Neurological signs and localization of parietal/frontal MC/MEC.

Table S3. MRI findings in dogs with meningocele and meningoencephalocele (Case 22 is mentioned twice as it had an intranasal MEC and a parietal MC).

Table S4. Results of CSF analysis in dogs with abnormal CSF examination.

Table S5a. Treatment and outcome of dogs with intranasal (ethmoidal and frontal) MEC (case 22 excluded based on the concurrent parietal MC).

Table S5b. Treatment and outcome of dogs with parietal/frontal MC/MEC and with concurrent intranasal and parietal MC/MEC. 